\title{
Distributed Cooperative Data Relaying for Diversity in Impulse-Based UWB Ad-Hoc Networks
}

\author{
Shouhong Zhu, Kin K. Leung, and Anthony G. Constantinides
}

\begin{abstract}
In this paper we develop and investigate two families of efficient distributed cooperative data relaying schemes that can be adopted to forward data in the impulse-based ultra wide band wireless ad-hoc network composed of a pair of source and destination and multiple parallel two-hop relays. The new schemes combine the mechanism of the medium access control and physical layers in a cooperative and distributed way to either select the best relay from multiple available ones for data forwarding or optimally combine the synchronized data forwarding of all participating relays, such as to improve the data transfer diversity. For distributed cooperative routing, we propose efficient protocols for use at all relays to perform an enhanced ultra wide band pulse sensing multiple access with the backoff periods deterministically and optimally mapped from associated instantaneous source-relay-destination route qualities, to ensure that only the best relay can firstly and successfully forward its received data to the destination; For distributed cooperative beamforming, we propose efficient protocols for use at all relays to take advantages of the widely spread and independently distributed multiple paths of the fading ultra wide band channels in the synchronized data forwarding combination, to create an optimally combined route for source-destination data transfer. Performance analysis and simulation studies show the effectiveness and efficiency of our proposed schemes.
\end{abstract}

Index Terms-Impulse-based UWB, wireless ad-hoc network, distributed cooperative data relaying, routing, beamforming.

\section{INTRODUCTION}

$\mathbf{T}$ HE recently permitted unlicensed use of the regulated ultra wide band (UWB) radio spectrum has been greatly propelling wireless ad-hoc networks with various applications in the environments where it is hard or not feasible to set up any infrastructure for communications [1][2]. To further investigate efficient transmission strategies for use in these UWB wireless ad-hoc networks, many research projects, including the EU-funded PULSERS project in which the authors of this paper are now participating, have been launched [3][4]. Motivated by the considerations listed as follows, in this paper we put our research focus on the development and investigation of efficient distributed cooperative data relaying for diversity in the impulse-based UWB wireless ad-hoc network composed of a pair of source and destination and multiple parallel two-hop relays: First, due to the broadcasting

Manuscript received February 4, 2008; revised June 15, 2008; accepted August 4, 2008. The associate editor coordinating the review of this paper and approving it for publication was R. Mallik.

The authors are with the Communications and Signal Processing Group, Department of Electrical and Electronic Engineering, Imperial College, London SW7 2BT, UK (e-mail: \{s.zhu, kin.leung, a.constantinides $\} @$ imperial.ac.uk). S. Zhu is the corresponding author.

This work was supported by the EU funded PULSERS research project. The contents of this paper were presented partially at IEEE ICC'2007, Glasgow, UK, June, 2007 and PIMRC'2007, Athens, Greece, September, 2007.

Digital Object Identifier 10.1109/TWC.2009.080163 nature of the media, a wireless ad-hoc network normally adopts a cooperative transmission strategy or another when forwarding data from a source to its destination via multiple available routes. The main idea is to forward data through an optimally selected or combined route from these available ones such that the communication resources can be utilized efficiently to achieve an overall performance with higher reliability, lower transmit power, and/or higher transmission rate [5][6]. Second, for the typical wireless ad-hoc network that involves multiple parallel two-hop relays, simple cooperative data relaying schemes usually include the route/relay selection based schemes, thus also called as the cooperative routing schemes [7][8][9], as well as the synchronized data forwarding combination based schemes, thus also called as the cooperative beamforming schemes [10]. These two families of cooperative data relaying schemes need to be implemented by cross-layer optimized protocols in a distributed way. Third, for the low data rate applications the UWB wireless ad-hoc networks more likely adopt the cheap and promising impulse-based UWB air-interfaces [3]. However, due to the strict regularization on the unlicensed use of the UWB radio spectrum as well as the unique characteristics of the impulse-based UWB data communications, the aforementioned distributed cooperative data relaying schemes face considerable challenges when applied. And their impulse-based UWB versions must undergo changes for effectiveness and efficiency.

Recent studies on distributed cooperative routing mainly focused on the narrow band cases and feasible and efficient supporting techniques enabling the data forwarding to be exclusively performed by the best relay [7][8]. In [7] a decentralized routing scheme was proposed, in which all relays adopt the carrier sensing multiple access (CSMA) with the route quality-related backoff periods as their medium access control (MAC) protocol. The backoff period adopted for each relay is chosen approximately adversely proportional to its associated route quality, but added with some degree of randomness to compensate the discreteness of the mapped back-off period to decrease the probability of the collisions with other participating relays. Such introduced randomness, however, is not necessary, without which the mapping can be greatly improved. In [8], the opportunistic routing concept was proposed, and pairs of request-to-send (RTS) and clearto-send (CTS) signal/message exchange among the source and destination as well as relays were introduced to enable collision avoidance and link quality measurements. Such RTSCTS exchange also results in unnecessary control information exchange burden, and again the mapping adopted can be further improved. Moreover, these two proposed schemes, 
when applied to the impulse-based UWB case in particular, are not straightforward. For example, the CSMA mechanism must be replaced by a suitable UWB pulse sensing multiple access (PSMA) mechanism, but it would be a big challenge to develop such a pulse sensing algorithm for quick medium activity check.

Recent studies on cooperative beamforming mainly focused on the narrow band cases with centralized implementations. These proposed schemes, when applied for distributed implementations as well as to the impulse-based UWB cases, are not straightforward. Fortunately, as the fading UWB channels exhibit widely spread and independently distributed multiple paths, the impulse-based UWB version of cooperative beamforming is actually feasible without severe mutual suppression on the transmitted signals of participating relays, and can be easily implemented in a distributed way. This is significantly different from that in the narrow band cases where the channel phases, in addition to the amplitudes, must be known at all participating relays to achieve the coherent combination of these synchronized data forwarding.

Our contributions in this paper can be summarized as below:

For distributed cooperative routing, we develop a group of protocols for use at all relays to perform an enhanced UWB PSMA with the backoff periods deterministically and optimally mapped from associated instantaneous (block-sense) source-relay-destination route qualities, to ensure that only the best relay can firstly and successfully forward data to the destination. For such protocols, we propose two UWB pulse sensing algorithms for quick medium activity check; prove that under mild conditions the uniformly distributed back-off periods can achieve minimum collision probability; discuss the tradeoff between the assigned acceptable collision probability and the resultant average data forwarding delay; and devise the methods that can be used to optimally represent the source-relay-destination route quality at relays. Performance analysis and simulation studies evidently show that our proposed distributed cooperative routing schemes, when with as little as 1-bit feedback on relay-destination link quality from the destination to relays, can achieve full diversity, and when without such feedback still exhibit improvements in performance as compared to the random routing scheme.

For distributed cooperative beamforming, we develop a group of protocols for use at all relays to optimally combine the synchronized data forwarding of participating relays by taking advantages of the widely spread and independently distributed multiple paths of the fading UWB channels. For such protocols, we analyze the data transfer performance under different data forwarding policies, and propose simple methods for distributed implementations. Performance analysis and simulation studies show that our proposed distributed cooperative beamforming schemes, without any feedback, still can achieve full diversity.

The rest of this paper is organized as follows: The network scenario under investigation and the signal model to be used are given in Section II. The developed efficient distributed cooperative routing schemes and their supporting techniques are presented in Section III. The developed efficient distributed cooperative beamforming schemes and their supporting techniques are presented in Section IV. Simulation studies are conducted in Section V. And finally the paper is concluded in Section VI.

\section{Network Scenario And Signal Model}

\section{A. Network Scenario}

Under investigation is an impulse-based UWB ad-hoc network composed of a pair of source and destination nodes and multiple available relay nodes located in between, thus there exist multiple parallel two-hop relaying routes from source to destination, in addition to the direct transmission (due to the limited space in this paper, here we do not include the direct transmission in our research, as such inclusion is straightforward). All these nodes are equipped with the impulse-based UWB air-interfaces and single-antennas, and all relays work in a half-duplex manner, i.e. each of these relays can either transmit or receive signal but cannot perform both at the same time.

\section{B. Impulse-Based UWB Data Link}

In the impulse-based UWB data communications, every information-bearing data symbol is conveyed by $N_{f}$ consecutive data-modulated ultra-short pulses, each with unit energy and effective width $T_{p}$, one per frame duration $T_{f}>>T_{p}$. The data symbol thus has energy $N_{f}$ and lasts $N_{f} T_{f}=T_{s}$. To smooth the resultant transmit spectrum of the UWB signal such as to comply with the requirements for the permitted unlicensed use, the pseudo-random time-hopping $(\mathrm{TH})$ or direct-sequence (DS) codes are often employed: TH codes shift the pulse positions from frame to frame at multiples of the chip duration say $T_{c}=T_{f} / 2>T_{p}$, while DS codes modify the pulse amplitudes (actually signs for binary values). Letting $c_{k} \in[0,1]$ and $a_{k} \in[-1,1]$ denote the TH and DS codes during the $k$-th frame, the transmitted symbol-long waveform, if without data modulation, can be represented as

$$
p_{T}(t)=\sum_{k=0}^{N_{f}-1} p\left(t-k T_{f}-c_{k} T_{c}\right)
$$

or

$$
p_{T}(t)=\sum_{k=0}^{N_{f}-1} a_{k} p\left(t-k T_{f}\right)
$$

where $p(t)$ is the waveform of the ultra-short pulse. If data are modulated by the pulse position modulation (PPM) or pulse amplitude modulation (PAM), the transmitted signal is

$$
s(t)=\sum_{i} p_{T}\left(t-i T_{s}-\frac{1+s_{i}}{2} \delta\right)
$$

or

$$
s(t)=\sum_{i} s_{i} p_{T}\left(t-i T_{s}\right)
$$

where $s_{i} \in[-1,1]$ is the transmitted data symbol at time index $i$, and $\delta>T_{p}$ is a small time delay used for the PPM. It should be noted that although the transmitted data symbols are normally assumed binary, it is straightforward to be extended to the case analogue values are adopted to take advantage of the channel capacity. For example the perfectly channel coded data symbols are to approach the channel capacity. In this 
paper we consider both the coded data symbol and uncoded binary data symbol cases.

As it is straightforward to be extended to any other case, here we only consider the case with DS codes and PAM data modulation, just for simplicity. If the transmit power is $P$ and the channel is $h(t)$ with maximum effective delay spread $T_{h}$ (in case $T_{f}$ is designed to be longer than $T_{h}$ then there would be no inter frame interference), the received signal is therefore

$$
r(t)=\sqrt{P} \sum_{i} s_{i} p_{R}\left(t-i T_{s}\right)+w(t)
$$

where $w(t)$ is the received noise assumed to be zero-mean Gaussian distributed, with power spectral density $N_{o}$, and

$$
\begin{gathered}
p_{R}(t)=p_{T}(t) * h(t)=\sum_{k=0}^{N_{f}-1} a_{k} p_{h}\left(t-k T_{f}\right) \\
p_{h}(t)=p(t) * h(t) .
\end{gathered}
$$

The received signal, after the pulse matched filter and Nyquist rate sampler, can be written as

$$
\begin{aligned}
& \int_{0}^{T_{s a}} r(t) p\left(t-i T_{s}-k T_{f}-j T_{s a}\right) d t=r_{i, k, j} \\
& \quad=s_{i} \sqrt{P} a_{k} h\left(j T_{s a}\right)+n_{i, k, j}
\end{aligned}
$$

where $T_{s a}$ is the Nyquist sampling interval, $j=0, \ldots, \frac{T_{f}}{T_{s a}}-1$, $k=0, \ldots, N_{f}-1, i=0, \ldots$, and $n_{i, k, j}$ is the corresponding zero-mean and $\sigma_{n}^{2}$-variance Gaussian noise item with $\sigma_{n}^{2}=$ $\frac{N_{o}}{2 T_{s a}}$. If a coherent receiver is adopted then $s_{i}$ can be detected based on the following decision variable

$$
\begin{aligned}
r_{i} & =\sum_{k=0}^{N_{f}-1} \sum_{j=0}^{\frac{T_{f}}{T_{s a}}-1} r_{i, k, j} a_{k} h\left(j T_{s a}\right) \\
& =s_{i} N_{f} \sqrt{P} \sum_{j=0}^{\frac{T_{f}}{T_{s a}}-1}\left|h\left(j T_{s a}\right)\right|^{2}+\sum_{k=0}^{N_{f}-1} \sum_{j=0}^{\frac{T_{f}}{T_{s a}}-1} n_{i, k, j} a_{k} h\left(j T_{s a}\right) .
\end{aligned}
$$

The signal to noise ratio (SNR) of $s_{i}$ in $r_{i}$, i.e. the receiver SNR of the impulse-based UWB data link, is

$$
\rho=\frac{P N_{f} \sum_{j}\left|h\left(j T_{s a}\right)\right|^{2}}{\sigma_{n}^{2}} .
$$

The above coherent receiver usually has a high complexity. For feasibility, many complexity-reduced receivers were proposed, these include various correlation-based receivers such as the ones based on energy detection, transmit reference, and, for the reciprocally equal channels, transmit time reversal [11][12]. The performance degradation in these complexityreduced receivers as compared with the coherent receiver is caused by the unavoided noise-times-noise item as well as the doubled signal-times-noise items in the calculated decision variable. With suitable improving techniques such as the multisymbol detection, all these complexity-reduced receivers can closely approach, with a fixed gap, to the coherent receiver in performance, especially in the high-SNR range. It is reasonable to take the coherent receiver as the baseline receiver in our research, as this paper mainly focuses on the distributed cooperative schemes designed for diversity improvements.

\section{Fading UWB Channel and Data Transfer Performance Measures}

Due to the ultra wide band property and the reflective transmission environments, UWB signals usually suffer from multiple delay-resolvable paths which appear as rays in clusters [13][14][15][16]. The basic model for the fading UWB channels, i.e. the Saleh-Valenzuela model [17], uses five parameters to describe these clustered rays: $m_{c}, \gamma_{c}, \lambda_{c}, \Gamma_{c}, \Lambda_{c}$. In this paper we just use such basic model, as many existing models can be regarded as its variants.

Generally there are two performance metrics that are used to characterize the data transfer over a fading channel: the overall bit error rate (BER) in the final data detection, and the probability of the outages in which the link can not support a given data rate.

Given receiver SNR $\rho$, the BER for the uncoded binary data symbols is

$$
\epsilon(\rho)=\mathrm{Q}(\sqrt{\rho})
$$

where $\mathrm{Q}(\cdot)$ is the error function. The overall or average BER for the data transfer over a fading channel is

$$
\epsilon_{\text {overall }}=\int \epsilon(\rho) p(\rho) d \rho
$$

where $p(\rho)$ is the probability density function (pdf) of the link receiver SNR.

Given data rate $r_{\rho_{o}}$ ( $\rho_{o}$ is the minimum SNR that can support data rate $r_{\rho_{o}}$ ), the outage probability for the coded data symbols is

$$
P_{\text {out }}=\operatorname{Pr}\left\{r<r_{\rho_{o}}\right\}=\operatorname{Pr}\left\{\rho<\rho_{o}\right\}=\operatorname{Pr}\left\{\frac{\rho}{\bar{\rho}}<\frac{\rho_{o}}{\bar{\rho}}\right\}
$$

where $\bar{\rho}$ is the average link receiver SNR. When $\bar{\rho}$ tends to infinity the asymptotic outage probability (AOP) exhibits the data transfer diversity order at high SNR region. Usually the data transfer AOP can be approximated as

$$
P_{\text {out }}^{(a s y)} \approx \Phi\left(\frac{\rho_{o}}{\bar{\rho}}\right)^{\omega}
$$

where $\omega$ is the diversity order, $\Phi$ is the corresponding gain/loss. It is clear that the diversity order depends only on the behavior of the pdf of normalized link receiver SNR $\frac{\rho}{\bar{\rho}}$ near the origin, as discussed in [18].

With the S-V model for the fading UWB channels, the normalized link receiver SNR exhibits a complicated distribution which is very difficult to derive. Here we just give an approximate relationship between the data transfer diversity order and the parameters of the fading UWB channel (with extensive simulations we found that the diversity order is approximately determined by $m_{c}$ as well as $\Gamma_{c} \Lambda_{c}+\gamma_{c} \lambda_{c}$, as given in [19])

$$
\omega \approx m_{c}\left[1+2\left(\gamma_{c} \lambda_{c}+\Gamma_{c} \Lambda_{c}\right)\right] .
$$

\section{EFFiCient Distributed CoOperative Routing SCHEMES}

\section{A. Cooperative Routing: Impulse-Based UWB Version}

Cooperative routing, as mentioned earlier, refers to the operation in which the best relay, associated with the highest instantaneous (block-sense) source-relay-destination route 
quality, is selected from multiple available ones for data forwarding. For its impulse-based UWB version we have the following remark:

Provided the impulse-based $U W B$ data relaying routes each exhibits data transfer AOP $\Phi\left(\frac{\rho_{o}}{\bar{\rho}}\right)^{\omega}$, the selected route from $M$ available ones according to the cooperative routing scheme exhibits data transfer $A O P$

$$
\left[\Phi\left(\frac{\rho_{o}}{\bar{\rho}}\right)^{\omega}\right]^{M}=\Phi^{M}\left(\frac{\rho_{o}}{\bar{\rho}}\right)^{\omega M} .
$$

This is obvious because the outage probability of the selected route is

$$
\begin{aligned}
P_{\text {out }} & =\operatorname{Pr}\left\{\rho_{m}<\rho_{o}, \quad m=1, \cdots, M\right\} \\
& =\operatorname{Pr}\left\{\rho_{1}<\rho_{o}\right\} \cdots \operatorname{Pr}\left\{\rho_{M}<\rho_{o}\right\}
\end{aligned}
$$

where the independence among routes $m=1, \cdots, M$ is utilized.

This remark demonstrates that with the impulse-based version of cooperative routing the diversity order of the data transfer can be increased.

\section{B. Distributed Implementation: Framework}

The distributed implementation of cooperative routing generally requires that the best relay knows it is the best one meanwhile any other relay knows it is not. Such a requirement, however, implies that each relay must know the order or rank information on the qualities of all routes, thus results in considerable channel side information exchange. An alternative that can be used to significantly reduce such channel side information exchange is as follows: all relays perform a CSMAlike MAC with the backoff periods satisfying that the higher the instantaneous (block-sense) source-relay-destination route quality the shorter the corresponding backoff period, such that the best relay firstly starts its data forwarding and any other relay aborts upon sensing the medium activity. Therefore each relay only needs to know the qualities of its own incoming and outgoing links. This is the concept of the decentralized opportunistic routing [7][8]. To suit the impulse-based UWB case, the distributed cooperative routing scheme as well as its supporting techniques can be devised within the following framework:

In the first time slot/hop all relays synchronize to the source, estimate their source-relay link qualities and acquire (if possible) their relay-destination link qualities, calculate their source-relay-destination route qualities, and then deterministically map them to their corresponding backoff periods according to the proposed mapping method, all in an independent manner. In the second time slot/hop, each relay monitors the medium activity via the proposed impulse-based UWB pulse sensing method, meanwhile counts down its backoff period from a commonly synchronized time instant: when the backoff period is counted down to zero, starts its data forwarding; if sensing that the medium is occupied by any other relay for data forwarding, aborts its prepared but not-yetstarted data forwarding (to deal with the hidden relay problem, a quick acknowledge signal is also designed to send back from the destination upon sensing the signal from any relay). We can see that by combining the mechanism of the MAC and physical layers in such a distributed way the developed distributed cooperative routing schemes can ensure that the best relay/route is efficiently selected.

In the following subsections, we discuss in detail the necessary supporting techniques for realizing the above distributed implementation framework.

\section{UWB Pulse Sensing for Quick Medium Activity Check}

In the proposed distributed cooperative routing schemes the quick medium activity check via UWB pulse sensing plays an important role. We know in checking whether or not such an event is present a suitable tool is the constant false alarm rate (CFAR) test [20], in which to produce a strong decision variable from weak UWB pulses for the CFAR test, a straightforward method is to synchronize the UWB signal first and obtain the data symbol decision variable. Such processing, however, needs a time-consuming synchronization, which affects the overall performance. In [21], an alternative that can be used to avoid synchronization was proposed. It extracts the spectral ray corresponding to the frame repetition period. Such processing, however, needs extra hardware and the independence among multiple paths severely weakens the spectral rays.

Here we propose an algorithm that does not need any synchronization but can carry out efficient UWB pulse sensing. We know that even without synchronization we still can obtain $\frac{T_{f}}{T_{s a}}-1$ groups of received signal samples $r_{i, k, j}, k=$ $0, \ldots, N_{f}-1, i=0, \ldots, M-1$ that are repeated by frame duration $T_{f}$. Based on each group of such samples, we can obtain

$$
D_{j}=\frac{1}{M} \sum_{i, k}\left|r_{i, k, j}\right|, \quad j
$$

and then

$$
\mathbf{d}=\left[D_{1} \cdots D_{j} \cdots D_{\frac{T_{f}}{T_{s a}}-1}\right]^{T} .
$$

We consider two options for the decision variable for the CFAR test: One is

$$
D=\|\mathbf{d}\|^{2}
$$

and the other is

$$
D=\max _{j} D_{j}
$$

where we utilize that the UWB channel has multiple paths with significantly different amplitudes. We refer these two algorithms as algorithm I and algorithm II respectively. From the simulations later we can see that algorithm II has a much better performance than algorithm I.

\section{Collision Probability, Optimum Mapping and Average Data Forwarding Delay}

In the proposed distributed cooperative routing schemes the collision probability is not zero as long as a non-zero period of time is needed to sense the UWB signal. To minimize such collision probability, below we discuss the optimum mapping from route qualities to backoff periods.

Provided the period of time needed to sense the UWB signal is $\Delta$, then the collision probability for $M$ relays is

$$
p_{c}=1-\int_{0}^{T} M f(x)[1-F(x+\Delta)]^{M-1} d x
$$


where $f(\cdot)$ is the pdf of the distribution of the mapped back-off periods, $F(\cdot)$ is the cumulative function of $f(\cdot)$. The second term is the probability without collision which corresponds to the case the shortest backoff period is shorter than other backoff periods by $\Delta$. We have following theorem.

Theorem 1. Provided the period of time needed to sense the UWB signal is $\Delta$ and $\Delta<<T$, the minimum collision probability, in the case with two participating relays, can be achieved by the uniformly distributed mapped back-off periods, and satisfies $\lim _{\frac{T}{\Delta} \rightarrow \infty} p_{c} \frac{T}{\Delta}=2$.

Proof: See Appendix A.

For the case with more than two participating relays, the optimum mapping is difficult to derive. But if the uniform distribution is also adopted, the corresponding collision probability can be known according to the following theorem.

Theorem 2. Provided the period of time needed to sense the $U W B$ signal is $\Delta$ and $\Delta<<T$, if the uniform distribution is adopted in the mapping, then the corresponding collision probability satisfies $\lim _{\frac{T}{\Delta} \rightarrow \infty} p_{c} \frac{T}{\Delta}=M$.

Proof: See Appendix B.

According to these two theorems, if $\Delta$ and the maximum acceptable collision probability are known, we can determine the corresponding $T$.

The resultant average data relaying delay, on the other hand, can be calculated if the mapped distribution is known

$$
T_{a d}=\int_{0}^{T} x g(x) d x
$$

where $g(\cdot)$ is the pdf of the distribution of the shortest back-off period

$$
g(x)=M f(x)[1-F(x)]^{M-1} .
$$

If the mapped back-off periods exhibit uniform distribution in support range $[0, \mathrm{~T}]$, then we have

$$
T_{a d}=\frac{T}{M+1} .
$$

Therefore we have following tradeoff between the resultant/acceptable average data relaying delay and acceptable/resultant collision probability

$$
T_{a d} p_{c}=\frac{M}{M+1} \Delta .
$$

\section{E. Route Quality Representation at Relays}

At each relay, the incoming source-relay link quality can be estimated based on the data symbol decision variable, by corresponding estimation algorithms such as the ones proposed in [22]. As for the outgoing relay-destination link quality acquired at each relay, we consider two options: the one with 1-bit feedback from the destination and the one without such feedback.

1) With 1-Bit Feedback: Totally there are three parameters to be set: threshold $\eta$, lower representation value $\eta_{l}$, and higher representation value $\eta_{h}$. The destination estimates the receiver SNR of each relay-destination link, and compares it with the threshold. If higher than the threshold, then the destination sends a ' 1 ' to the associated relay, otherwise sends a ' 0 '. The relay, upon receiving a ' 1 ', treats the receiver SNR of its relaydestination link quality as $\eta_{h}$; otherwise, a ' 0 ' will cause the relay to approximate it by $\eta_{l}$, i.e.

$$
\widehat{\rho}_{m d}= \begin{cases}\eta_{l}, & \rho_{m d}<\eta \\ \eta_{h}, & \rho_{m d}>\eta\end{cases}
$$

In practical implementations, the values of these three parameters can be optimized to achieve the best overall performance.

The following theorem demonstrates the data transfer performance of the proposed distributed cooperative routing scheme over fading UWB channels for the coded data symbols when adopting the decode and forward policy.

Theorem 3. Provided the impulse-based UWB data links each exhibits data transfer $A O P \Phi\left(\frac{\rho_{o}}{\bar{\rho}}\right)^{\omega}$, for the coded data symbols when adopting the decode and forward policy the source-relay and 1-bit relay-destination link qualities-selected route from two available ones exhibits data transfer AOP $4 \Phi^{2}\left(\frac{\rho_{o}}{\bar{\rho}}\right)^{2 \omega}$.

Proof: See Appendix C.

This theorem demonstrates that the proposed scheme, when with as little as 1-bit feedback, can achieve full diversity, and the corresponding overall performance is close to that with full precision feedback (the same as that of perfect cooperative routing).

2) Without Feedback: When there is no such feedback, the relay is simply selected based on the incomding source-relay link quality only. The following theorem demonstrates the data transfer performance of the proposed distributed cooperative routing scheme over fading UWB channels for the coded data symbols when adopting the decode and forward policy.

Theorem 4. Provided the impulse-based UWB data links each exhibits data transfer $A O P \Phi\left(\frac{\rho_{o}}{\bar{\rho}}\right)^{\omega}$, for the coded data symbols when adopting the decode and forward policy the source-relay link quality-selected route from two available ones exhibits data transfer $A O P \Phi\left(\frac{\rho_{o}}{\bar{\rho}}\right)^{\omega}$.

Proof: See Appendix D.

This theorem demonstrates that the proposed scheme, when without feedback, has no diversity gain, but exhibits improved overall performance as compared with the random routing scheme. For example when each link receiver SNR exhibits the exponential distribution (corresponding to the case with singlepath Rayleigh channels) the corresponding data transfer AOP is $\Phi \frac{\rho_{o}}{\bar{\rho}}$ whereas for the random routing scheme it is $2 \Phi \frac{\rho_{o}}{\bar{\rho}}$, with a $3 \mathrm{~dB}$ gap in performance.

\section{EfFicient Distributed Cooperative BEAMFORMING SCHEMES}

\section{A. Cooperative Beamforming: Impulse-Based UWB Version}

Cooperative beamforming, as mentioned earlier, refers to the operation in which all participating relays synchronize to the source and forward their received data, with suitable weights, to the destination to create an optimally combined route for source-destination data transfer. Thanks to the widely spread and independently distributed multiple paths of the fading UWB channels, the impulse-based UWB version of 
such cooperative beamforming is actually feasible without severe mutual suppression on the transmitted signals from participating relays, thus has the potential to achieve full diversity.

The received signal at the destination, when there are two relays, can be represented as

$$
\begin{aligned}
r(t) & =q_{1} \sqrt{P} \widetilde{s}^{(1)} \sum_{k=0}^{N_{f}-1} a_{k} p_{h_{1}}\left(t-k T_{f}\right) \\
& +q_{2} \sqrt{P} \widetilde{s}^{(2)} \sum_{k=0}^{N_{f}-1} a_{k} p_{h_{2}}\left(t-k T_{f}\right)+w(t)
\end{aligned}
$$

where

$$
\widetilde{s}^{(m)}=\frac{s \sqrt{\rho_{s m}}+n_{m}}{\sqrt{\rho_{s m}+1}}, \quad n_{m} \in N(0,1), \quad m=1,2
$$

for the amplify and forward policy,

$$
\widetilde{s}^{(m)}= \begin{cases}s, & \text { if } \rho_{s m} \geq \rho_{o} \\ 0, & \text { otherwise }\end{cases}
$$

for the coded data symbols when adopting the decode and forward policy, and

$$
\widetilde{s}^{(m)}=\left\{\begin{array}{l}
s, \text { with probability } 1-\epsilon_{s m} \\
-s, \text { otherwise }
\end{array}\right.
$$

for the uncoded binary data symbols when adopting the decode and forward policy; $q_{1}$ and $q_{2}$ are the weights satisfying $q_{1}^{2}+$ $q_{2}^{2}=1$, the constraint on total transmit power. As $p_{h_{1}}(t)$ and $p_{h_{2}}(t)$, the received UWB pulses distorted by the channels from two relays, are almost uncorrelated, the receiver SNRs of the combined routes for different data forwarding policies can be derived respectively.

It should be noted that because the case of $q_{m}=1, m \in$ $(1,2)$ corresponds to a route selector, the cooperative beamforming scheme is no worse in performance than the cooperative routing scheme. This also implies that the cooperative beamforming scheme can achieve full diversity.

1) Amplify and Forward Policy: The matched function in the corresponding coherent detection should be a scaled version of

$$
\begin{aligned}
& q_{1} \frac{\sqrt{\rho_{s 1}}}{\sqrt{\rho_{s 1}+1}} \sum_{k=0}^{N_{f}-1} a_{k} p_{h_{1}}\left(t-k T_{f}\right) \\
& +q_{2} \frac{\sqrt{\rho_{s 2}}}{\sqrt{\rho_{s 2}+1}} \sum_{k=0}^{N_{f}-1} a_{k} p_{h_{2}}\left(t-k T_{f}\right)
\end{aligned}
$$

so the combined route has the receiver SNR as follows

$$
\rho_{a a f}=\frac{\left(\frac{q_{1}^{2} \rho_{s 1} \rho_{1 d}}{\rho_{s 1}+1}+\frac{q_{2}^{2} \rho_{s 2} \rho_{2 d}}{\rho_{s 2}+1}\right)^{2}}{\rho_{s 1}\left(\frac{q_{1}^{2} \rho_{1 d}}{\rho_{s 1}+1}\right)^{2}+\rho_{s 2}\left(\frac{q_{2}^{2} \rho_{2 d}}{\rho_{s 2}+1}\right)^{2}+\frac{q_{1}^{2} \rho_{s 1} \rho_{1 d}}{\rho_{s 1}+1}+\frac{q_{2}^{2} \rho_{s 2} \rho_{2 d}}{\rho_{s 2}+1}} .
$$

The optimum set of weights for relays can be derived by assuming the derivatives of the resultant SNR with respect to the weights be zero. For relay 1 such obtained weight satisfies

$$
q_{1}^{2}=\frac{\left(\frac{\rho_{s 2} \rho_{2 d}}{\rho_{s 2}+1}\right)^{2}-\frac{\rho_{s 1} \rho_{1 d}}{\rho_{s 1}+1} \frac{\rho_{s 2} \rho_{2 d}}{\rho_{s 2}+1}\left(1+2 \frac{\rho_{2 d}}{\rho_{s 2}+1}\right)}{\left(\frac{\rho_{s 1} \rho_{1 d}}{\rho_{s 1}+1}\right)^{2}+\left(\frac{\rho_{s 2} \rho_{2 d}}{\rho_{s 2}+1}\right)^{2}-2 \frac{\rho_{s 1} \rho_{1 d} d}{\rho_{s 1}+1} \frac{\rho_{s 2} \rho_{2 d}}{\rho_{s 2}+1}\left(1+\frac{\rho_{1 d}}{\rho_{s 1}+1}+\frac{\rho_{2 d}}{\rho_{s 2}+1}\right)} .
$$

It should be noted that this obtained weight may be outside $[0,1]$ in which case the weight can be set by comparing with the cases of $q_{m}=1, m=1,2$ where

$$
\rho_{a a f}=\frac{\rho_{s m} \rho_{m d}}{\rho_{s m}+\rho_{m d}+1} \text {. }
$$

2) Coded Data Symbols and Decode and Forward Policy: The combined route has the receiver SNR as follows

$$
\rho_{d a f 1}=\left\{\begin{array}{l}
q_{1}^{2} \rho_{1 d}+q_{2}^{2} \rho_{2 d}, \quad \text { if } \rho_{s 1} \geq \rho_{o}, \rho_{s 2} \geq \rho_{o} \\
q_{1}^{2} \rho_{1 d}, \quad \text { if } \rho_{s 1} \geq \rho_{o}, \rho_{s 2}<\rho_{o} \\
q_{2}^{2} \rho_{2 d}, \quad \text { if } \rho_{s 1}<\rho_{o}, \rho_{s 2} \geq \rho_{o} \\
0, \quad \text { otherwise }
\end{array}\right.
$$

The optimum set of weights for relays are

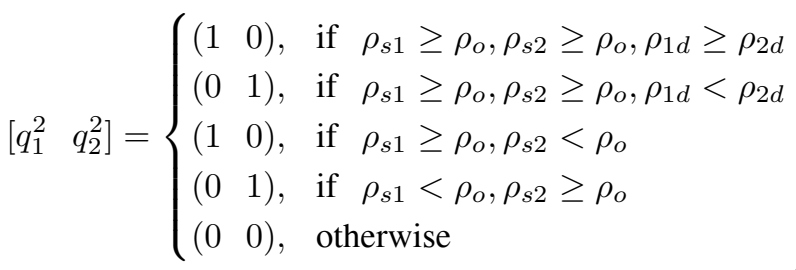

3) Uncoded Binary Data Symbols and Decode and Forward Policy: The matched function in the corresponding coherent detection should be a scaled version of

$$
\begin{aligned}
& \left(1-2 \epsilon_{s 1}\right) q_{1} \sum_{k=0}^{N_{f}-1} a_{k} p_{h_{1}}\left(t-k T_{f}\right) \\
& +\left(1-2 \epsilon_{s 2}\right) q_{2} \sum_{k=0}^{N_{f}-1} a_{k} p_{h_{2}}\left(t-k T_{f}\right)
\end{aligned}
$$

where $\epsilon_{s 1}$ and $\epsilon_{s 2}$ are the BERs of the links from the source to relay 1 and relay 2 . When two relays both correctly or incorrectly detect the uncoded binary data symbols the combined route has the receiver SNR as follows

$$
\rho_{\text {daf } 2}^{A}=\left|\frac{\left(1-2 \epsilon_{s 1}\right) q_{1}^{2} \rho_{1 d}+\left(1-2 \epsilon_{s 2}\right) q_{2}^{2} \rho_{2 d}}{\sqrt{\left(1-2 \epsilon_{s 1}\right)^{2} q_{1}^{2} \rho_{1 d}+\left(1-2 \epsilon_{s 2}\right)^{2} q_{2}^{2} \rho_{2 d}}}\right|^{2}
$$

otherwise it is

$$
\rho_{\text {daf } 2}^{B}=\left|\frac{\left(1-2 \epsilon_{s 1}\right) q_{1}^{2} \rho_{1 d}-\left(1-2 \epsilon_{s 2}\right) q_{2}^{2} \rho_{2 d}}{\sqrt{\left(1-2 \epsilon_{s 1}\right)^{2} q_{1}^{2} \rho_{1 d}+\left(1-2 \epsilon_{s 2}\right)^{2} q_{2}^{2} \rho_{2 d}}}\right|^{2} .
$$

So the resultant overall BER at the destination is

$$
\begin{aligned}
& \epsilon_{d a f 2}=\left(1-\epsilon_{s 1}-\epsilon_{s 2}+\epsilon_{s 1} \epsilon_{s 2}\right) \mathrm{Q}\left(\sqrt{\rho_{d a f 2}^{A}}\right) \\
& +\epsilon_{s 1} \epsilon_{s 2}\left[1-\mathrm{Q}\left(\sqrt{\rho_{d a f 2}^{A}}\right)\right]+x
\end{aligned}
$$

where $x$, when $\left(1-2 \epsilon_{s 1}\right) q_{1}^{2} \rho_{1 d}>\left(1-2 \epsilon_{s 2}\right) q_{2}^{2} \rho_{2 d}$, is

$$
\begin{aligned}
& x=\left(\epsilon_{s 2}-\epsilon_{s 1} \epsilon_{s 2}\right) \mathrm{Q}\left(\sqrt{\rho_{d a f 2}^{B}}\right) \\
& +\left(\epsilon_{s 1}-\epsilon_{s 1} \epsilon_{s 2}\right)\left[1-\mathrm{Q}\left(\sqrt{\rho_{d a f 2}^{B}}\right)\right]
\end{aligned}
$$

otherwise it is

$$
\begin{aligned}
& x=\left(\epsilon_{s 1}-\epsilon_{s 1} \epsilon_{s 2}\right) \mathrm{Q}\left(\sqrt{\rho_{d a f 2}^{B}}\right) \\
& +\left(\epsilon_{s 2}-\epsilon_{s 1} \epsilon_{s 2}\right)\left[1-\mathrm{Q}\left(\sqrt{\rho_{d a f 2}^{B}}\right)\right]
\end{aligned}
$$

Here it is difficult to derive the optimum set of weights. 


\section{B. Distributed Implementation: A Simple Solution}

The distributed implementation of the cooperative bean forming schemes generally requires the weights for relays $i$ forwarding their received data to the destination. We consider simple solution: equal weights. This requires a mild conditic that each relay knows the total number of the participatir relays. The following theorem demonstrates the correspondir data transfer performance of the proposed distributed coope ative beamforming scheme over fading UWB channels for th coded data symbols when adopting the decode and forwal policy.

Theorem 5. Provided the impulse-based UWB data links eac exhibits data transfer AOP $\Phi\left(\frac{\rho_{o}}{\bar{\rho}}\right)^{\omega}$, if the widely spread an independently distributed multipath channels for different lini are uncorrelated from each other, then the combined rou. adopting the proposed distributed cooperative beamformir. scheme with equal weights exhibits the data tranfer AOP less than

$$
\left(4^{\omega}+2^{\omega+1}+1\right) \Phi^{2}\left(\frac{\rho_{o}}{\bar{\rho}}\right)^{2 \omega}
$$

Proof: See Appendix E.

This theorem implies the proposed scheme can achieve full diversity.

\section{Simulation Studies}

The proposed two families of distributed cooperative data relaying schemes and their supporting techniques are evaluated by simulation studies. In the simulations, we assume the following settings: the UWB ultra-short pulses have the waveform as the second derivative of the Gaussian function, with effective width $0.7 \mathrm{~ns}$; a data symbol duration has 16 frames, each frame lasts 16 times of pulse width; data are modulated by the PAM; the UWB channels are set as IEEE Standard UWB channel CM-1 with parameters $(\Lambda, \Gamma, \lambda, \gamma)$ as $(0.047,22.61,0.15,12.53)$ and all rays in the channels exhibit Nakagami- $m_{c}=0.5$ distribution, which implies that such impulse-based UWB link has data transfer AOP

$$
\Phi\left(\frac{\rho_{o}}{\bar{\rho}}\right)^{\omega} \approx 25\left(\frac{\rho_{o}}{\bar{\rho}}\right)^{3.4}
$$

and on average two pulses disturbed by independently produced UWB channels with the above parameters are almost uncorrelated.

\section{A. Distributed Cooperative Routing}

Fig. 1 shows the performance of the proposed CFAR testbased UWB pulse sensing algorithms. We can see that to achieve miss probability 0.001 the proposed algorithm II with CFAR 0.001 only needs 8 data symbols at $15 \mathrm{~dB}$ link receiver SNR. It has a better performance than algorithm I, this is because the multiple paths in the fading UWB channel exhibit significantly different amplitudes. It has only around $3 \mathrm{~dB}$ gap in performance as compared with the algorithm based on the synchronized unknown data symbols.

Fig. 2 shows the data transfer performance of the proposed distributed cooperative routing schemes over fading UWB

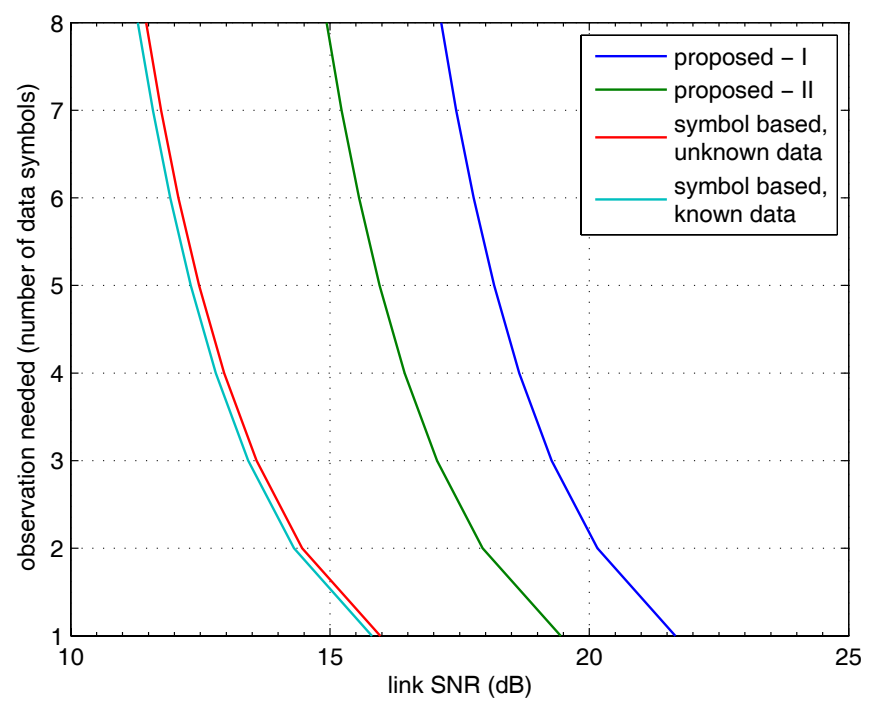

Fig. 1. The performance of the proposed CFAR test-based UWB pulse sensing algorithms: Observation length needed to achieve miss probability 0.001 at CFAR 0.001 .

channels for different data forwarding policies and different data symbol formats. We can see that for all cases the proposed schemes, when with as little as 1-bit feedback, can achieve full diversity, and when without such feedback exhibit performance improvements.

\section{B. Distributed Cooperative Beamforming}

Fig. 3 shows the data transfer performance of the proposed distributed cooperative beamforming schemes over fading UWB channels for different data forwarding policies and different data symbol formats. From Fig. 3a and $3 b$ we can see that for the amplify and forward policy the cooperative beamforming schemes have better performance than the cooperative routing schemes; the proposed schemes, without feedback, still can achieve full diversity with the overall performance very close to that of the cooperative routing schemes. From Fig. $3 \mathrm{c}$ we can see that for the decode and forward policy and coded data symbols the proposed scheme can achieve full diversity with the overall performance a small gap to that of the cooperative routing or beamforming scheme. From Fig. $3 \mathrm{~d}$ we can see that for the decode and forward policy and uncoded binary data symbols the proposed scheme exhibits significant diversity improvements.

\section{CONCLusions}

In this paper we developed and investigated two families of efficient distributed cooperative data relaying schemes that can be adopted to forward data within an impulse-based UWB ad-hoc network composed of a pair of source and destination and multiple parallel two-hop relays. Performance analysis and simulation studies evidently show the effectiveness and efficiency of our proposed schemes: The proposed distributed cooperative routing schemes, when with as little as 1-bit feedback can achieve full diversity, and when without such feedback still exhibit improvements as compared to the random routing scheme; The proposed distributed cooperative beamforming schemes can achieve full diversity with overall 


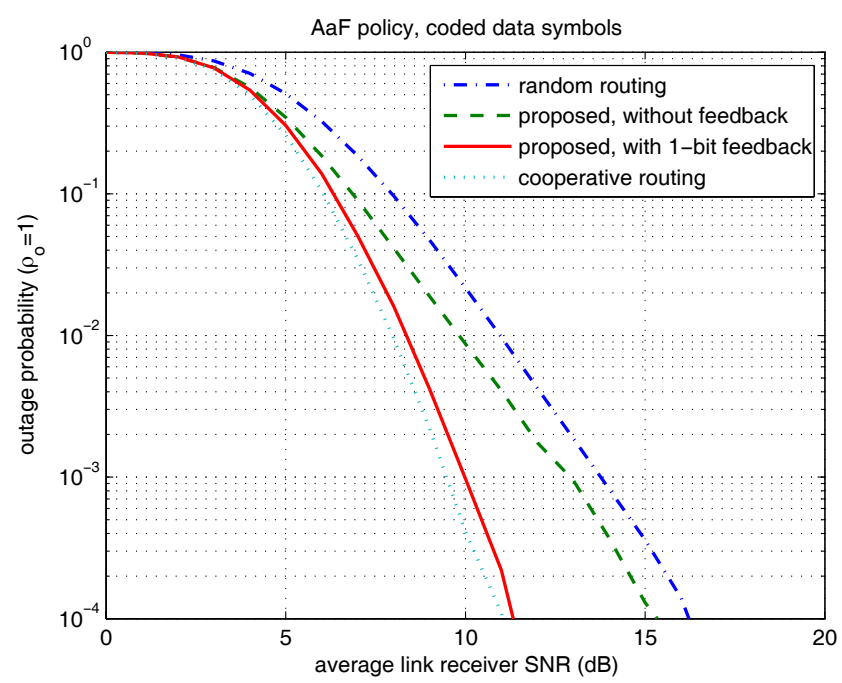

(a)

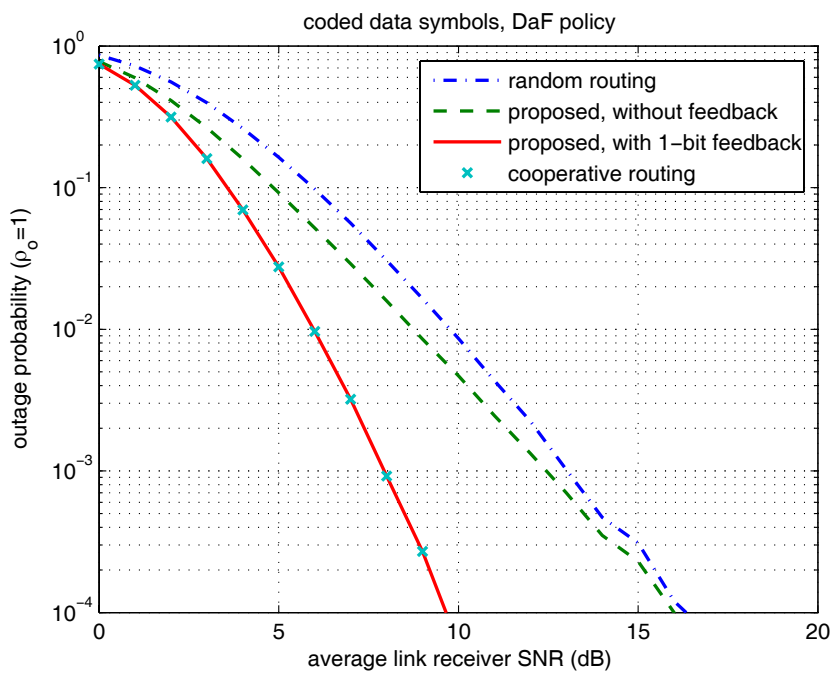

(c)

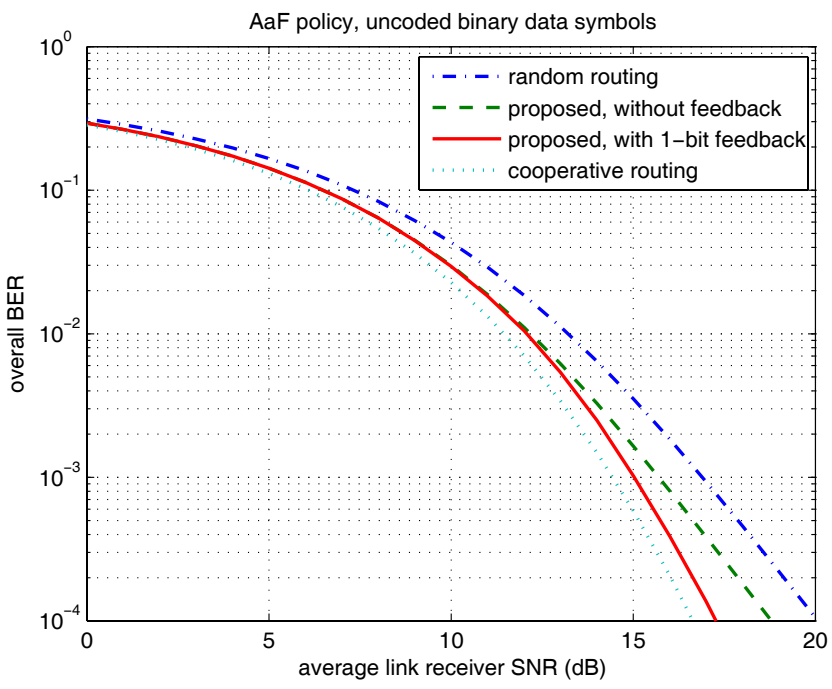

(b)

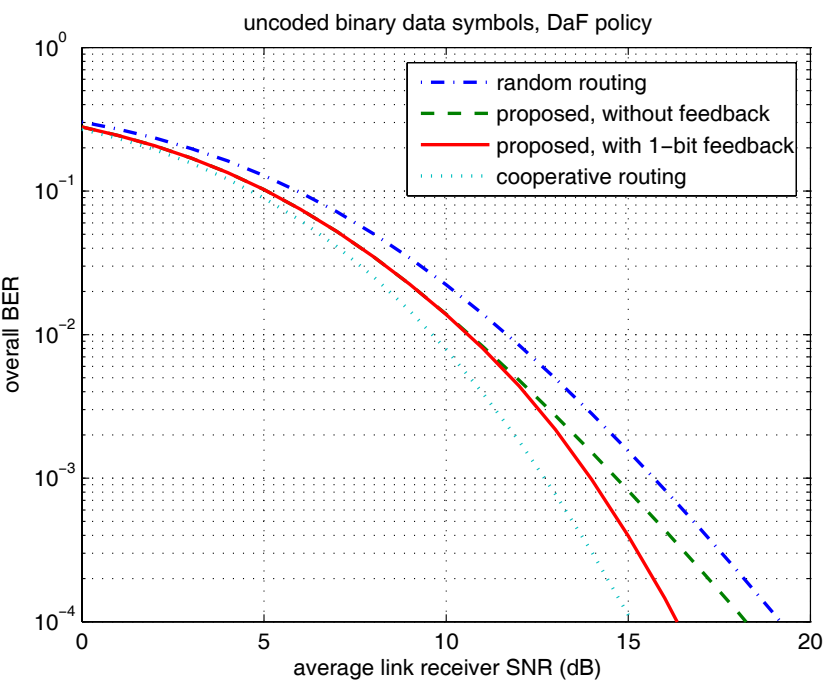

(d)

Fig. 2. The data transfer performance of the proposed distributed cooperative routing schemes over fading UWB channels: (a) the amplify and forward policy and coded data symbols; b) the amplify and forward policy and uncoded binary data symbols; (c) the coded data symbols and decode and forward policy; d) the uncoded binary data symbols and decode and forward policy.

performance just a small gap to that of perfect cooperative routing.

\section{APPENDIX}

\section{A. Proof of Theorem 1}

Proof: The collision probability is

$$
\begin{aligned}
p_{c} & =1-\int_{0}^{T} M f(x)[1-F(x+\Delta)]^{M-1} d x \\
& \int_{0}^{T} M f(x)\left\{[1-F(x)]^{M-1}-[1-F(x+\Delta)]^{M-1}\right\} d x
\end{aligned}
$$

where we utilize

$$
\int_{0}^{T} M f(x)[1-F(x)]^{M-1} d x=1 .
$$

When $M=2$, we have

$$
p_{c}=2 \int_{0}^{T} f(x)[F(x+\Delta)-F(x)] d x .
$$

Further, when $\frac{T}{\Delta}$ tends to $\infty, p_{c}$ tends to

$$
2 \Delta \int_{0}^{T} f^{2}(x) d x .
$$

To minimize $p_{c}$ subject to

$$
\int_{0}^{T} f(x) d x=1
$$

$f(x)$ must be the pdf of the uniform distribution.

If

$$
f(x)=\frac{1}{T}, \quad x \in[0, T]
$$

then we have

$$
\lim _{\frac{T}{\Delta} \rightarrow \infty} p_{c} \frac{T}{\Delta}=2 .
$$

This proves the theorem. 


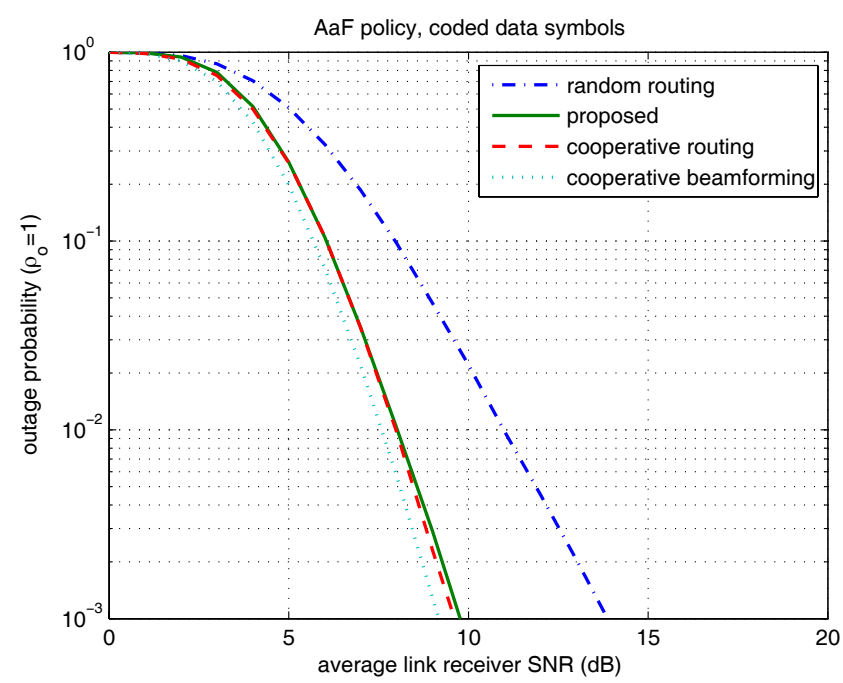

(a)

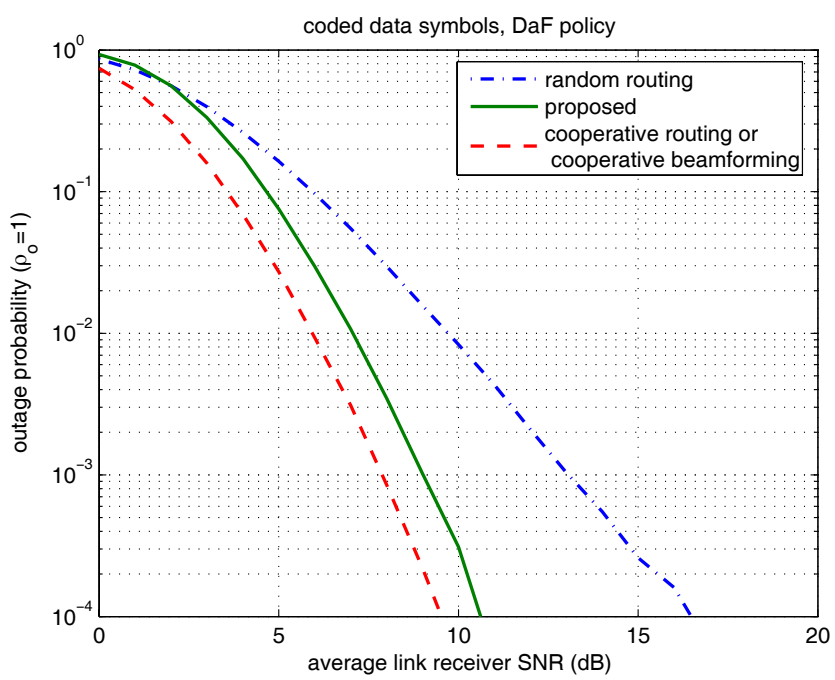

(c)

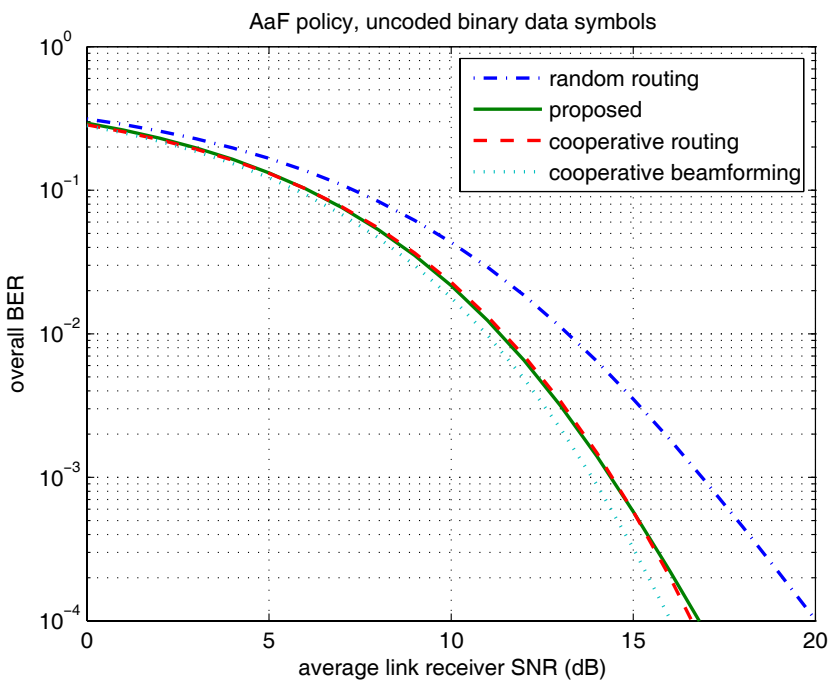

(b)

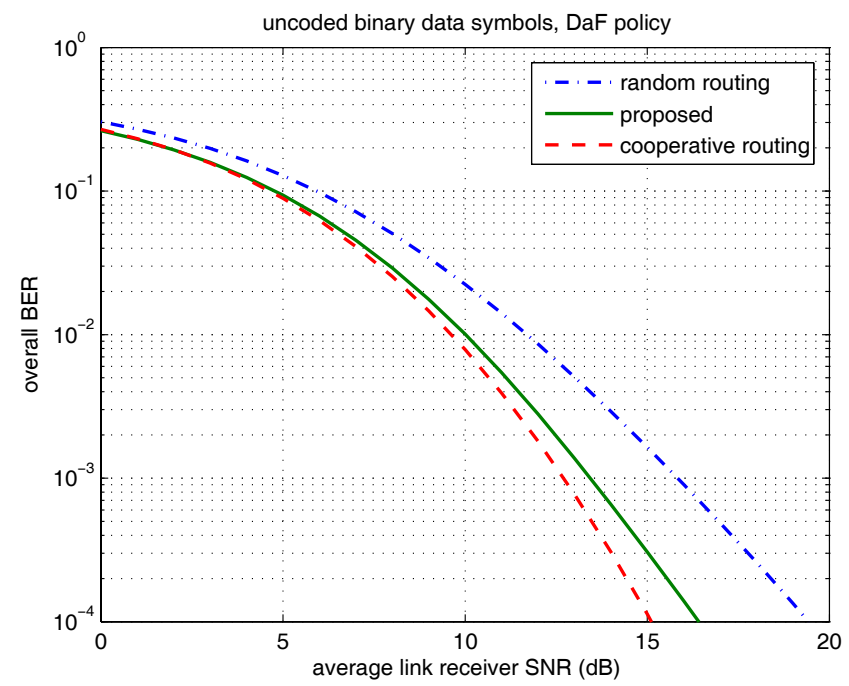

(d)

Fig. 3. The data transfer performance of the proposed distributed cooperative beamforming schemes over fading UWB channels: (a) the amplify and forward policy and coded data symbols; b) the amplify and forward policy and uncoded binary data symbols; (c) the coded data symbols and decode and forward policy; d) the uncoded binary data symbols and decode and forward policy.

$$
p_{c}=\int_{0}^{T} M f(x)\left\{[1-F(x+\Delta)+F(x+\Delta)-F(x)]^{M-1}-[1-F(x+\Delta)]^{M-1}\right\} d x .
$$

\section{B. Proof of Theorem 2}

Proof: Continued from the proof of Theorem 1, for $M>$ 2 we get (51) (at the top of the next page). Further, when $\frac{T}{\Delta}$ tends to $\infty, p_{c}$ tends to

$$
M(M-1) \Delta \int_{0}^{T} f^{2}(x)[1-F(x)]^{M-2} d x .
$$

If the uniform distribution is adopted in the mapping, then we have

$$
\lim _{\frac{T}{\Delta} \rightarrow \infty} p_{c} \frac{T}{\Delta}=M
$$

This proves the theorem.

\section{Proof of Theorem 3}

Proof: The selected route has the receiver SNR at the destination as follows

$$
\rho_{\text {overall }}= \begin{cases}\min \left(\rho_{s 1}, \rho_{1 d}\right), & \text { if } \min \left(\rho_{s 1}, \widehat{\rho}_{1 d}\right)>\min \left(\rho_{s 2}, \widehat{\rho}_{2 d}\right) \\ \min \left(\rho_{s 2}, \rho_{2 d}\right), & \text { otherwise }\end{cases}
$$

where $\rho_{x y}$ refers to the receiver SNR of the link from $\mathrm{x}$ to $\mathrm{y}$ : 's' refers to the source, 'd' refers to the destination, ' 1 ' refers to relay 1 . So the outage probability of the selected route is

$$
\begin{aligned}
P_{\text {out }} & =\operatorname{Pr}\left\{\rho_{\text {overall }}<\rho_{o}\right\} \\
& =\operatorname{Pr}\left\{\min \left(\rho_{s 1}, \rho_{1 d}\right)<\rho_{o}, \min \left(\rho_{s 1}, \widehat{\rho}_{1 d}\right)>\min \left(\rho_{s 2}, \widehat{\rho}_{2 d}\right)\right\} \\
& +\operatorname{Pr}\left\{\min \left(\rho_{s 2}, \rho_{2 d}\right)<\rho_{o}, \min \left(\rho_{s 2}, \widehat{\rho}_{2 d}\right)>\min \left(\rho_{s 1}, \widehat{\rho}_{1 d}\right)\right\}
\end{aligned}
$$


There exist four independent cases

$$
\begin{aligned}
P_{\text {out }} & =P_{\text {out }}^{(1)} \operatorname{Pr}\left\{\widehat{\rho}_{1 d}>\rho_{o}, \widehat{\rho}_{2 d}>\rho_{o}\right\} \\
& +P_{\text {out }}^{(2)} \operatorname{Pr}\left\{\widehat{\rho}_{1 d}>\rho_{o}, \widehat{\rho}_{2 d}<\rho_{o}\right\} \\
& +P_{\text {out }}^{(3)} \operatorname{Pr}\left\{\widehat{\rho}_{1 d}<\rho_{o}, \widehat{\rho}_{2 d}>\rho_{o}\right\} \\
& +P_{\text {out }}^{(4)} \operatorname{Pr}\left\{\widehat{\rho}_{1 d}<\rho_{o}, \widehat{\rho}_{2 d}<\rho_{o}\right\} .
\end{aligned}
$$

When $\widehat{\rho}_{1 d}>\rho_{o}$ and $\widehat{\rho}_{2 d}>\rho_{o}$, we have

$$
\begin{aligned}
P_{\text {out }}^{(1)} & =\operatorname{Pr}\left\{\rho_{s 2}<\rho_{s 1}<\rho_{o}\right\}+\operatorname{Pr}\left\{\rho_{s 1}<\rho_{s 2}<\rho_{o}\right\} \\
& =\operatorname{Pr}\left\{\rho_{s 1}<\rho_{o}\right\} \operatorname{Pr}\left\{\rho_{s 2}<\rho_{o}\right\} .
\end{aligned}
$$

When $\widehat{\rho}_{1 d}>\rho_{o}$ and $\widehat{\rho}_{2 d}<\rho_{o}$, we have

$$
\begin{aligned}
P_{\text {out }}^{(2)} & =\operatorname{Pr}\left\{\rho_{s 2}<\rho_{s 1}<\rho_{o}\right\}+\operatorname{Pr}\left\{\rho_{s 2}<\rho_{o}, \rho_{s 1}<\rho_{o}\right\} \\
& +\operatorname{Pr}\left\{\rho_{2 d}<\rho_{o}, \rho_{s 1}<\rho_{o}\right\} \\
& -\operatorname{Pr}\left\{\rho_{s 2}<\rho_{o}, \rho_{s 1}<\rho_{o}, \rho_{2 d}<\rho_{o}\right\} .
\end{aligned}
$$

When $\widehat{\rho}_{1 d}<\rho_{o}$ and $\widehat{\rho}_{2 d}>\rho_{o}$, we have

$$
\begin{aligned}
P_{\text {out }}^{(3)} & =\operatorname{Pr}\left\{\rho_{s 1}<\rho_{o}, \rho_{s 2}<\rho_{o}\right\}+\operatorname{Pr}\left\{\rho_{s 1}<\rho_{o}, \rho_{1 d}<\rho_{o}\right\} \\
& -\operatorname{Pr}\left\{\rho_{s 1}<\rho_{o}, \rho_{s 2}<\rho_{o}, \rho_{1 d}<\rho_{o}\right\} \\
& +\operatorname{Pr}\left\{\rho_{s 1}<\rho_{s 2}<\rho_{o}\right\} .
\end{aligned}
$$

When $\widehat{\rho}_{1 d}<\rho_{o}$ and $\widehat{\rho}_{2 d}<\rho_{o}$, we have

$$
\begin{aligned}
P_{\text {out }}^{(4)} & =\operatorname{Pr}\left\{\rho_{s 1}<\rho_{o}\right\}+\operatorname{Pr}\left\{\rho_{1 d}<\rho_{o}\right\} \\
& -\operatorname{Pr}\left\{\rho_{s 1}<\rho_{o}, \rho_{1 d}<\rho_{o}\right\} \\
& +\operatorname{Pr}\left\{\rho_{s 2}<\rho_{o}\right\}+\operatorname{Pr}\left\{\rho_{2 d}<\rho_{o}\right\} \\
& -\operatorname{Pr}\left\{\rho_{s 2}<\rho_{o}, \rho_{2 d}<\rho_{o}\right\} .
\end{aligned}
$$

So the corresponding data transfer AOP can be simplified as

$$
\begin{aligned}
P_{\text {out }}^{(\text {asy })} & =\Phi^{2}\left(\frac{\rho_{o}}{\bar{\rho}}\right)^{2 \omega}+\frac{1}{2} \Phi^{2}\left(\frac{\rho_{o}}{\bar{\rho}}\right)^{2 \omega}+\frac{1}{2} \Phi^{2}\left(\frac{\rho_{o}}{\bar{\rho}}\right)^{2 \omega} \\
& +2 \Phi^{2}\left(\frac{\rho_{o}}{\bar{\rho}}\right)^{2 \omega}+O\left[\left(\frac{\rho_{o}}{\bar{\rho}}\right)^{\omega}\right]
\end{aligned}
$$

where $O\left[\left(\frac{\rho_{o}}{\bar{\rho}}\right)^{\omega}\right]$ is the sum of the terms with higher orders. This proves the theorem.

\section{Proof of Theorem 4}

Proof: The selected route has the receiver SNR at the destination as follows

$$
\rho_{\text {overall }}= \begin{cases}\min \left(\rho_{s 1}, \rho_{1 d}\right), & \text { if } \rho_{s 1}>\rho_{s 2} \\ \min \left(\rho_{s 2}, \rho_{2 d}\right), & \text { otherwise }\end{cases}
$$

So the outage probability of the selected route is

$$
\begin{aligned}
P_{\text {out }} & =\operatorname{Pr}\left\{\rho_{\text {overall }}<\rho_{o}\right\} \\
& =\operatorname{Pr}\left\{\rho_{s 1}<\rho_{o}, \rho_{s 1}>\rho_{s 2}\right\}+\operatorname{Pr}\left\{\rho_{1 d}<\rho_{o}, \rho_{s 1}>\rho_{s 2}\right\} \\
& -\operatorname{Pr}\left\{\rho_{s 1}<\rho_{o}, \rho_{1 d}<\rho_{o}, \rho_{s 1}>\rho_{s 2}\right\} \\
& +\operatorname{Pr}\left\{\rho_{s 2}<\rho_{o}, \rho_{s 2}>\rho_{s 1}\right\}+\operatorname{Pr}\left\{\rho_{2 d}<\rho_{o}, \rho_{s 2}>\rho_{s 1}\right\} \\
& -\operatorname{Pr}\left\{\rho_{s 2}<\rho_{o}, \rho_{2 d}<\rho_{o}, \rho_{s 2}>\rho_{s 1}\right\}
\end{aligned}
$$

The second and fifth items are

$$
\operatorname{Pr}\left\{\rho_{1 d}<\rho_{o}\right\} \operatorname{Pr}\left\{\rho_{s 1}>\rho_{s 2}\right\}=\frac{1}{2} \operatorname{Pr}\left\{\rho_{1 d}<\rho_{o}\right\}
$$

$$
\operatorname{Pr}\left\{\rho_{2 d}<\rho_{o}\right\} \operatorname{Pr}\left\{\rho_{s 2}>\rho_{s 1}\right\}=\frac{1}{2} \operatorname{Pr}\left\{\rho_{2 d}<\rho_{o}\right\}
$$

The sum of the first and forth items is

$$
\operatorname{Pr}\left\{\rho_{s 1}<\rho_{o}, \rho_{s 2}<\rho_{o}\right\}=\operatorname{Pr}\left\{\rho_{s 1}<\rho_{o}\right\} \operatorname{Pr}\left\{\rho_{s 2}<\rho_{o}\right\}
$$

The third and sixth items are

$$
\begin{aligned}
& \operatorname{Pr}\left\{\max \left(\rho_{s 1}, \rho_{s 2}\right)<\rho_{o}\right\} \operatorname{Pr}\left\{\rho_{1 d}<\rho_{o}\right\} \\
& \operatorname{Pr}\left\{\max \left(\rho_{s 1}, \rho_{s 2}\right)<\rho_{o}\right\} \operatorname{Pr}\left\{\rho_{2 d}<\rho_{o}\right\}
\end{aligned}
$$

So the corresponding data transfer AOP can be simplified as

$$
P_{\text {out }}^{(\text {asy })}=\frac{1}{2} \Phi\left(\frac{\rho_{o}}{\bar{\rho}}\right)^{\omega}+\frac{1}{2} \Phi\left(\frac{\rho_{o}}{\bar{\rho}}\right)^{\omega}+O\left[\left(\frac{\rho_{o}}{\bar{\rho}}\right)^{\omega}\right] .
$$

This proves the theorem.

\section{E. Proof of Theorem 5}

Proof: The resultant receiver SNR at the destination is

$$
\rho=\left\{\begin{array}{l}
0.5 \rho_{1 d}+0.5 \rho_{2 d}, \quad \text { if } \rho_{s 1} \geq \rho_{o}, \rho_{s 2} \geq \rho_{o} \\
0.5 \rho_{1 d}, \quad \text { if } \rho_{s 1} \geq \rho_{o}, \rho_{s 2}<\rho_{o} \\
0.5 \rho_{2 d}, \quad \text { if } \rho_{s 2} \geq \rho_{o}, \rho_{s 1}<\rho_{o} \\
0, \quad \text { otherwise }
\end{array}\right.
$$

so the corresponding outage probability is

$$
\begin{aligned}
& \operatorname{Pr}\left\{\rho<\rho_{o}\right\} \\
& \quad=\operatorname{Pr}\left\{\rho_{s 1} \geq \rho_{o}\right\} \operatorname{Pr}\left\{\rho_{s 2} \geq \rho_{o}\right\} \operatorname{Pr}\left\{0.5 \rho_{1 d}+0.5 \rho_{2 d}<\rho_{o}\right\} \\
& \quad+\operatorname{Pr}\left\{\rho_{s 1} \geq \rho_{o}\right\} \operatorname{Pr}\left\{\rho_{s 2}<\rho_{o}\right\} \operatorname{Pr}\left\{0.5 \rho_{1 d}<\rho_{o}\right\} \\
& \quad+\operatorname{Pr}\left\{\rho_{s 1}<\rho_{o}\right\} \operatorname{Pr}\left\{\rho_{s 2} \geq \rho_{o}\right\} \operatorname{Pr}\left\{0.5 \rho_{2 d}<\rho_{o}\right\} \\
& \quad+\operatorname{Pr}\left\{\rho_{s 1}<\rho_{o}\right\} \operatorname{Pr}\left\{\rho_{s 2}<\rho_{o}\right\}
\end{aligned}
$$

As

$\operatorname{Pr}\left\{0.5 \rho_{1 d}+0.5 \rho_{2 d}<\rho_{o}\right\} \leq \operatorname{Pr}\left\{0.5 \rho_{1 d}<\rho_{o}\right\} \operatorname{Pr}\left\{0.5 \rho_{2 d}<\rho_{o}\right\}$

and the impulse-based UWB data links each exhibits data transfer AOP $\Phi\left(\rho_{o} / \bar{\rho}\right)^{\omega}$, so the data transfer AOP is

$$
\begin{aligned}
P_{\text {out }}^{(\text {asy })} & \leq \Phi^{2} 4^{\omega}\left(\frac{\rho_{o}}{\bar{\rho}}\right)^{2 \omega}+\Phi^{2} 2^{\omega}\left(\frac{\rho_{o}}{\bar{\rho}}\right)^{2 \omega} \\
& +\Phi^{2} 2^{\omega}\left(\frac{\rho_{o}}{\bar{\rho}}\right)^{2 \omega}+\Phi^{2}\left(\frac{\rho_{o}}{\bar{\rho}}\right)^{2 \omega} \\
& =\left(4^{\omega}+2^{\omega+1}+1\right) \Phi^{2}\left(\frac{\rho_{o}}{\bar{\rho}}\right)^{2 \omega}
\end{aligned}
$$

This proves the theorem.

\section{REFERENCES}

[1] The UWB Forum, website: http://www.uwbforum.org.

[2] C. S. R. Murthy and B. S. Manoj, Ad-Hoc Wireless Networks: Architectures and Protocols. Prentice Hall, 2004.

[3] The EU funded PULSERS research project, website: http://www.pulsers.eu.

[4] The EU funded PULSERS research project at Imperial College, website: http://www.commsp.ee.ic.ac.uk/ wiser/pulsers/.

[5] J. N. Laneman, D. N. C. Tse, and G. W. Wornell, "Cooperative diversity in wireless networks: efficient protocols and outage behavior," IEEE Trans. Inform. Theory, vol. 51, no. 12, pp. 3062-3080, Dec. 2004.

[6] K. Azarian, H. El Gamal, and P. Schniter, "On the achievable diversityvs-multiplexing tradeoff in half-duplex cooperative channels," IEEE Trans. Inform. Theory, vol. 51, no. 12, pp. 4152-4172, Dec. 2005. 
[7] C. Akcaba, R. U. Nabar, and K. K. Leung, "A PHY/MAC approach to wireless routing," in Proc. IEEE ICC 2006.

[8] A. Bletsas, A. Khisti, D. P. Reed, and A. Lippman, "A simple cooperative diversity method based on network path selection," IEEE J. Select. Areas Commun., vol. 24, no. 3, pp. 659-672, Mar. 2006.

[9] S. Zhu and K. K. Leung, "Distributed cooperative routing for UWB ad-hoc networks," in Proc. IEEE ICC 2007, Glasgow, UK, May 2007.

[10] S. Zhu, K. K. Leung, and A. G. Constantinides, "Distributed cooperative data transfer for UWB ad-hoc network," in Proc. PIMRC2007, Greece, Sept. 2007.

[11] R. C. Qiu, C. Zhou, N. Guo, and J. Q. Zhang, "Time reversal with MISO for ultra-wideband communications: experimental results," IEEE Antennas and Wireless Propagation Lett., vol. 5, no. 1, pp. 269-273, Dec. 2006.

[12] N. Guo, B. M. Sadler, and R. C. Qiu, "Reduced-complexity UWB time reversal techniques and experiemental results," IEEE Trans. Wireless Commun., vol. 6, no. 12, pp. 4221-4226, Dec. 2007.

[13] R. Qiu, "A study of the ultrawideband wireless propagation channel and optimum UWB receiver design," IEEE J. Select. Areas Commun., vol. 20, no. 9, pp. 1628-1637, Dec. 2002.

[14] R. Qiu, "A generalized time domain multipath channel and its application in ultrawideband wireless optimal receiver design: system performance analysis," IEEE Trans. Wireless Commun., vol. 5, no. 10, pp. 2685-2695, Oct. 2004.

[15] D. Cassioli, M. Z. Win, and A. F. Molisch, "The ultra wide bandwidth indoor channel: from statistical model to simulations," IEEE J. Select. Areas Commun., vol. 20, no. 6, pp. 1247-1257, Aug. 2002.

[16] A. F. Molisch, "Ultrawideband propagation channels: theory, measurement, and modeling," IEEE Trans. Veh. Technol., vol. 54, no. 5, pp. 1528-1545, Sept. 2005.

[17] A. Saleh and R. Valenzuela, "A statistical model for indoor multipath propagation," IEEE J. Select. Areas Commun., vol. 5, no. 2, pp. 128-137, Feb. 1987.

[18] O. Dabeer and E. Masry, "Asymptotic behavior of the pairwise error probability with applications to transmit beamforming and Rake receivers," IEEE Trans. Wireless Commun., vol. 4, no. 2, pp. 444-452, Mar. 2005.

[19] S. Zhu, K. K. Leung, and A. G. Constantinides, "Multiple access, scheduling and routing for MAS type B," the EU-funded PULSERS project deliverable report, May, 2008.

[20] H. Rohling, "Radar CFAR thresholding in clutter and multiple target situations," IEEE Trans. Aerospace and Electronic Syst., vol. 19, no. 4, pp. 608-621, July 1983.

[21] N. J. August, H. J. Lee, and D. S. Ha, "Enabling distributed medium access control for impulse based ultra wide band radios," IEEE Trans. Veh. Technol., vol. 56, no. 3, pp. 1064-1075, May 2007.

[22] S. Im and E. J. Powers, "An algorithm for estimating signal-to-noise ratio of UWB signals," IEEE Trans. Veh. Technol., vol. 54, no. 5, pp. 1905-1908, Sept. 2005.

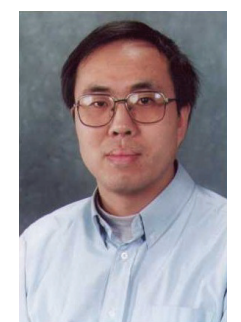

Shouhong Zhu (M'97) received his B.Eng.Sc. and M.Eng.Sc. degrees in electronic engineering from Northwestern Telecommunication Engineering Institute (now Xidian University), xi'an, China in 1985 and 1988 respectively, his $\mathrm{PhD}$ degree in time keeping and disseminating from Chinese Academy of Sciences, China in 1996, and his Ph.D. degree in communications and signal processing from Imperial College, London, UK in 2005. He joined Chinese Academy of Sciences in 1988. On leave from there, from 1999 he spent one year at the Communications and Signal Processing Group, Imperial college, London, UK as a visiting scholar. Since then, he has been studying and working in the same group. He is currently a Research Associate. His interests include signal processing and its applications. His current interests include signal processing in wireless communication networks, cooperative transmission strategies, cross-layer protocol design and evaluation, etc.

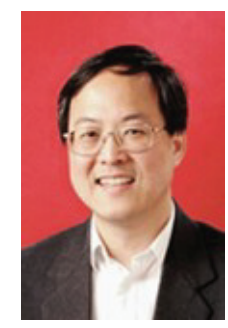

Kin K. Leung (F'01) received his BS degree from the Chinese University of Hong Kong in 1980, and his MS and Ph.D. degrees in computer science from University of California, Los Angeles, in 1982 and 1985, respectively. He joined AT\&T Bell Labs in 1986 and worked at its successor companies, AT\&T Labs and Bell Labs of Lucent Technologies, until 2004. Since then, he has been the Tanaka Chair Professor in Internet Technology at Imperial College London. His research interests include network resource allocation, MAC protocol, TCP/IP protocol, mobility management, network architecture, real-time applications and teletraffic issues for wireless IP networks, sensor and ad-hoc networks. $\mathrm{He}$ is also interested in a wide variety of wireless technologies, including IEEE 802.11, 802.16, and 3G and future generation cellular networks.

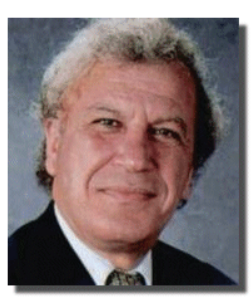

Anthony G. Constantinides (S68M74SM78F98) is the Professor of Signal Processing at Imperial College London, London, U.K. He has been actively involved with research in various aspects of digital filter design, digital signal processing, and communications for more than 30 years. His recent work has been directed toward the demanding signal processing problems arising from the various areas of telecommunication. This work is supported by research grants and contracts from various government and industrial organizations. He has published several books and papers in learned journals in the area of digital signal processing and its applications.

Dr. Constantinides is a Fellow of the Royal Academy of Engineering and the Institution of Electrical Engineers (IEE) and an honorary member of Eta Kappa $\mathrm{Nu}$. He has served as the First President of the European Association for Signal Processing (EURASIP). He has been on, and is currently serving as, a member of many technical program committees of the IEEE, the IEE, and other international conferences. He organized the first ever international series of meetings on Digital Signal Processing, in London initially in 1967 and in Florence since 1972. In 1985, he was awarded the French National Honor of Chevalier, Palmes Academiques, and in 1996 was elevated to Officier, Palmes Academiques. He holds honorary doctorates from European and Far Eastern universities, several visiting professorships, distinguished lectureships, fellowships, and other honors around the world. He served as a Member of the Board of Governors of the IEEE Signal Processing Society and a member of several Technical Committees of the IEEE and the IEE, and he is on the Editorial Board of the ProceEdings of THE RoyAl Society and on several professional journals. 